\title{
Research Paper \\ Exercises of Lumbar Stabilizer Muscles, Resistance Training, and Soy Food Consumption: A Comparative Study Between Old and Young Women
}

\author{
*Mahsa Rastegar Moghadam Mansouri ${ }^{1}$, Amir Hossein Haghighi ${ }^{1}$, Roya Askari ${ }^{1}$
}

1. Department of Exercise Physiology, Faculty of Physical Education and Sport Sciences, Hakim Sabzevari University, Sabzevar, Iran.

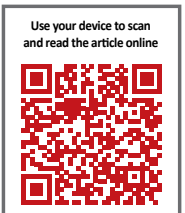

Crtation: Rastegar MMM, Haghighi AH, Askari R. [Exercises of Lumbar Stabilizer Muscles, Resistance Training, and Soy Food Consumption: A Comparative Study Between Old and Young Women (Persian)]. Iranian Journal of Ageing. 2017; 12(1):4455. http://dx.doi.org/10.21859/sija-120142

doi : http://dx.doi.org/10.21859/sija-120142

Received: 19 Sep. 2016 Accepted: 25 Nov. 2016

Key words: Aging, Soy milk, Paraspinal muscles

\begin{abstract}
A B S T R A C T
Objectives Aging and change in the skeletal muscle are certain challenges faced by the elderly. This condition leads to a decrease in the musculoskeletal functions, physical function, and disability compared to the younger individuals. Thus, the current study aimed at examining the exercise of back stabilizer muscles of aged women compared to the young ones in response to resistance training and soy milk supplementation.

Methods \& Materials The study had a quasi-experimental design conducted on 27 old women (Mean [SD] age: $69.60[3.16]$ years) and 10 young ones. The aged women were randomly divided into back stabilizer exercise plus soy milk, back stabilizer exercise, and control groups ( 9 subjects in each group). They took part in exercise-nutrition interventions for 10 weeks, 3 sessions per week. The subjects were given soy milk to drink three times in a day with and without exercise. Ultrasonic features of multifidus, ability, back performance, and nutrition status of aged women were measured pre- and post-intervention. Data were analyzed by 2-way ANOVA.

Results Mean (SD) multifidus cross-sectional area of aged women $\left(3.49[0.908] \mathrm{cm}^{2}\right)$ was significantly smaller than that of young ones $\left(7.42[1.52] \mathrm{cm}^{2}\right)$ during pre-intervention. Following the intervention, it significantly increased in both stabilizer exercise plus soy milk and back stabilizer exercise groups $(P<0.05$, $5.41[0.44]$ and $5.28[1.18] \mathrm{cm}^{2}$, respectively), but did not completely modify. Ability and back performance of intervention groups showed significant development compared to control group $(P<0.05$, $-10.2[3.3]$ and $-11.72[2.8] \mathrm{cm}^{2}$, respectively).

Conclusion Ten weeks of stabilizer exercise and consuming soy milk led to an increase in the ultrasonic features of back multifidus muscle of aged women and decreased the age-related muscle changes. On the other hand, improvement in multifidus muscles not only resulted in ability and back performance promotion, but also led to independency and social activity development in the aged women.
\end{abstract}

\section{Extended Abstract}

\section{Objectives}

ld age is associated with significant reduction in neuromuscular performance and atrophy of the lower extremities [1] more than the upper extremities [2, 3]. Appropriate diet and exercise are considered as strong remedies to deal with this corrosive trend $[4,5]$.

Sport activities and consumption of protein $(20 \mathrm{~g} / \mathrm{d}) \mathrm{im}$ prove muscle performance in old people [6], and the com-

\section{* Corresponding Author:}

Mahsa Rastegar Moghadam Mansouri, PhD

Address: Department of Exercise Physiology, Faculty of Physical Education and Sport Sciences, Hakim Sabzevari University, Sabzevar, Iran.

Tel: +98 (912) 0620717

E-mail: rastegar.moghadam.mansouri@gmail.com 
Table 1. Between-group investigation of the Multifidus muscle in young people $(n=10)$ and old people $(n=27)$ after intervention

\begin{tabular}{|c|c|c|c|c|c|}
\hline \multirow{2}{*}{ Group } & \multirow{2}{*}{$\begin{array}{c}\text { Statistics of Central Ten- } \\
\text { dency and Dispersion } \\
\text { Post Test (M } \pm \text { SD) }\end{array}$} & \multirow{2}{*}{$\begin{array}{l}\text { Multiple Comparisons of the Experi- } \\
\text { mental and Control Groups }\end{array}$} & \multicolumn{3}{|c|}{$95 \%$ of Confidence Interval for Differences } \\
\hline & & & $\begin{array}{l}\text { Mean of Differ- } \\
\text { ences }\end{array}$ & $\begin{array}{l}\text { Lower Ex- } \\
\text { tremity }\end{array}$ & $\begin{array}{l}\text { Upper } \\
\text { Extremity }\end{array}$ \\
\hline \multicolumn{6}{|c|}{ CSAAve $\left(\mathrm{cm}^{2}\right)$ (Index) } \\
\hline Young People=YP & $7.42 \pm 1.52$ & Exercise & $2.00 \pm 0.58+$ & 0.32 & 3.69 \\
\hline $\begin{array}{l}\text { Core stability } \\
\text { exercises }\end{array}$ & $5.41 \pm 0.44$ & Exercise+soy milk & $2.14 \pm 0.49 \dagger$ & 0.72 & 3.56 \\
\hline $\begin{array}{l}\text { Core stability } \\
\text { exercises }\end{array}$ & $5.28 \pm 1.18$ & Youth & & & \\
\hline +Soy milk & & Control & $3.81 \pm 0.368^{\dagger}$ & 2.74 & 4.88 \\
\hline Control & $3.61 \pm 1.15$ & & & & \\
\hline
\end{tabular}

ARAMEN IN ING

Abbreviations: CI: Confidence Interval; $\uparrow$ : represents a significant difference between groups using Bonferroni post-hoc test and assuming $\mathrm{P}<0.05$; M: Mean; SD: The Standard Deviation of desired group.

bination of these two interventions increases muscle protein synthesis [7]. Among the protein sources, soybean is considered as an economical source due to its essential amino acids and high absorption rate [8]. According to a survey, no study has investigated the exercises of lumbar stabilizer muscles in old women compared to young ones; therefore, this study aimed to examine the practice of lumbar stabilizer muscles on old women compared to young ones, following resistance training and consumption of soy protein.

\section{Methods \& Materials}

The current study was a quasi-experimental study involving two age groups. Statistical population comprised all women referred to clinics in three districts of Tehran. According to $\alpha=0.05$ and test power $=0.08$, the sample size was calculated to be 8 subjects in each group [9]. Old women were randomly assigned to lumbar stabilization exercises $(n=9)$, lumbar stabilization exercises plus soy milk $(n=9)$, and control $(n=9)$ groups. The training period lasted 10 weeks with three sessions of training per week. Written informed consent was obtained from all subjects $[10,11]$. Principles of the Declaration of Helsinki, comments and licenses (936-6049) of Research Ethics Committee and all ethical codes were observed. To examine the ability to practice stabilizing muscles of the lower back, 10 young women were selected. Ability $(\mathrm{ODI})^{1}$, back performance (BPS) ${ }^{2}$, nutritional status of older women, and ultrasonic profile of multifidus lumbar muscle were recorded for both age groups before and after the intervention. Soy milk was given 3 times a day $(186 \mathrm{~mL}$ in each meal) with and without exercise [12].

1. Oswestry Disability Index

2. Back performance scale
Lumbar stabilization exercises [13, 14] included boat, shoulder flexion in the horizontal axis, back to ground push-ups, sit-up, wheel pose, back extension (prone), hip flexion (supine), and shoulder flexion (standing). In order to respect the principle of overload, traband (training boat, shoulder flexion, hip flexion), free weights (sit-up, wheel pose, waist extension, shoulder flexion), and sphygmomanometer (back to ground push-up) were used and the intensity of exercise was increased while creating compatibility (Borg scale 8 or less). Cross-sectional area of multifidus muscle was examined in the fourth lumbar vertebra by sonography device (Italy) [14]. Data normalization was examined by Shapiro-Francia test, and the equality of variances was evaluated by Levene's test. The dependent variables before and after the intervention were analyzed with 2-way analysis of variance (ANOVA) by Stata. Bonferroni post hoc test was used to determine the differences between groups. Significance level was set at $\mathrm{P}<0.05$.

\section{Results}

Old women participating in the study had an average (SD) age of 69.60 (3.16) years, an average (SD) weight of 66.49 (3.18) kg, and their average (SD) body mass index was 25.58 (3.94) $\mathrm{kg} / \mathrm{m}^{2}$. The young women who participated in the study had an average (SD) age of 30.08 (4.1) years, an average (SD) weight of $66.28(6.1) \mathrm{kg}$, and their average (SD) body mass index was $25.78(1.6) \mathrm{kg} / \mathrm{m}^{2}$ before the intervention. Baseline characteristics had no significant change in any of the age groups $(\mathrm{P}<0.05)$.

In the between-group investigation of young and old people before the intervention, the average (SD) longitudinal vectors in young and old women were 23.05 (3.04) 
$\mathrm{mm}$ and $16.04(3.09) \mathrm{mm}$ (5.59 to 8.43, 7.01[0.71]), confidence interval $=95 \%$ ), respectively. Based on the average transverse vector, a significant difference was observed between young women $(33.77$ [5.11] $\mathrm{mm})$ and old ones (25.69 [5.16] mm), (5.7 to 10.44, 8.07[1.18], confidence interval $=95 \%$ ). The average (SD) cross-section of multifidus muscle in young women was equivalent to 7.42 $(1.52) \mathrm{cm}^{2}$, and for old women, it was equivalent to 3.49 $(0.908) \mathrm{cm}^{2}$ (3.35 to $4.49,0.28$ [3.92], confidence inter$\mathrm{val}=95 \%$ ). This significant difference was not observed in the average shape of multifidus muscle in comparison to young and old women groups $(-0.027$ to $0.056,0.0147$ $[0.02]$, confidence interval $=95 \%$ ).

Regarding the within-group investigation of young and old women after the intervention, a significant difference between the means of longitudinal vector, transverse vector, and shape of multifidus muscle of young women compared with old women in waist stabilizer training group and lumbar stabilization exercises plus soy milk was observed $(\mathrm{P}<0.05)$. But, despite the decline, the difference between the means of cross-sectional area of multifidus muscle in young women and old women was significant $(\mathrm{P}<0.05)$ (Table 1). In the within-group investigation, the average transect vector, transverse vector, and the crosssectional area of multifidus muscle increased significantly in both groups $(\mathrm{P}<0.05)$. The changes in the figure were not statistically significant $(\mathrm{P}=0.134)$. Table 1 . Betweengroup investigation of the multifidus muscle in young $(\mathrm{n}=10)$ and old women $(\mathrm{n}=27)$ after the intervention.

The capability in between-group investigation was improved significantly in both groups of core stability exercises plus soy milk $(\mathrm{P}=0.0001)$ and core stability exercises alone $(\mathrm{P}=0.003)$, compared to control group. Also in within-group investigation, the ability of the two interventional groups significantly improved $(\mathrm{P}<0.05)$. In between-group investigation, waist performance of waist stabilizer exercise plus soy milk significantly improved in two items $(\mathrm{P}<0.05)$. In within-group investigation, both groups showed significant improvement in the same two items $(\mathrm{P}<0.05)$.

In between-group investigation, the nutritional status of protein and fat received was significantly increased in waist stabilizer exercise plus soy milk group than that of the other two groups $(\mathrm{P}<0.05)$. In the withingroup investigation, only the protein received in waist stabilizing exercise plus soy milk group showed a significant increase $(\mathrm{P}<0.05)$.

\section{Conclusion}

In this study, multifidus muscle size, ability level, and waist performance were found to be improved. Numerous studies have been conducted in line with the present study [9] and improved capability and increased waist multifidus muscle cross-sectional area have been reported [9]. Old people respond to sports-dietary interventions slower than young people due to anabolic resistance and disruption of anabolic routes $[8,9,14]$. Hence, regular consumption of soy milk, along with exercise [8] and slow performance of exercises, improve proteolytic routes and muscular hypertrophy in old people [14]. Lack of soy milk group and the failure to perform exercises by young women were among the limitations of this study. Generally, 10 weeks of sports-nutrition intervention led to muscle improvement, performance, and capabilities of old women and reducing the difference between them and young women.

\section{Acknowledgments}

This research was extracted from the $\mathrm{PhD}$ thesis of the first author, in Hakim Sabzevari University, Sabzevar, Iran.

\section{Conflict of Interest}

The authors declared no conflicts of interest. 


\title{
تمرينيذيرى عضات ثثبيتكنثده كمرى زنان سالمند در مقايسه با زنان جوان به دنبال تمرين مقاومتى و مصرف يروتئين سويا
}

\author{
"مهسا رستكار مقدم منصورى'، اميرحسين حقيقى'، رويا عسكرى'
}

ا - كروه فيزيولوزي ورزشى، دانشكده علوم ورزشى، دانشكَاه حكيم سبزوارى، سبزوار، ايران.

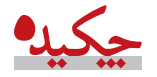

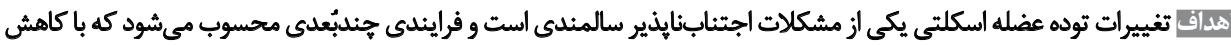

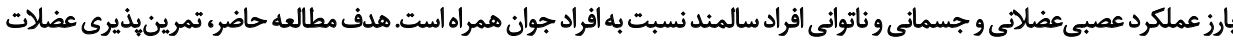

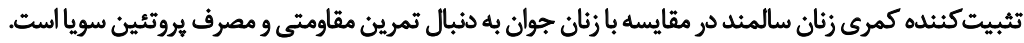

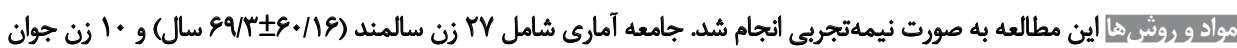

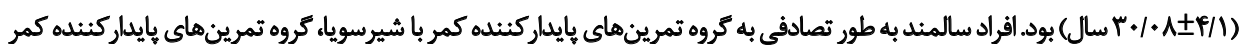

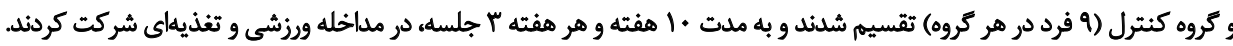

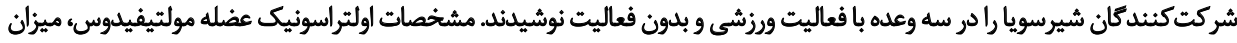

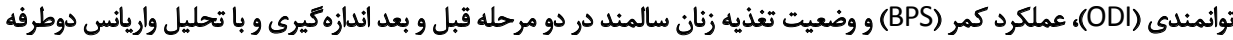

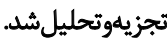

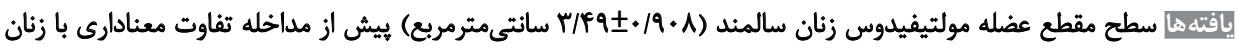

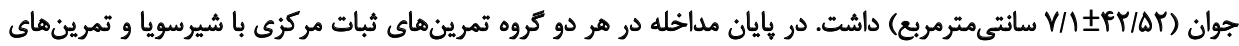

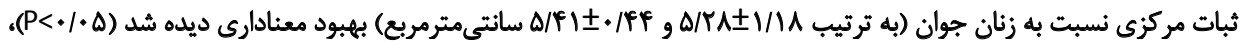

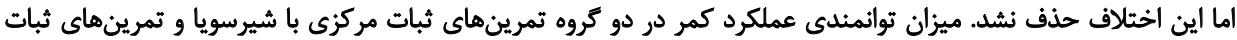

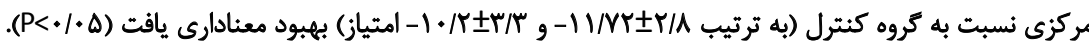

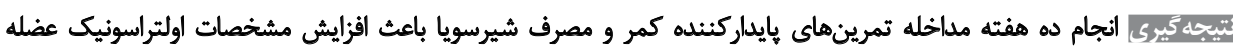

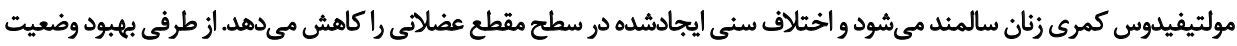

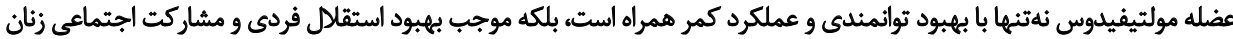

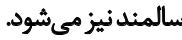

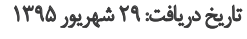
تاريخ يذيرش: ه• آذر هوبات

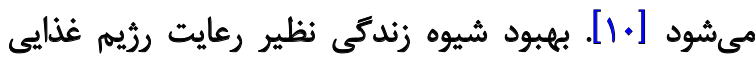

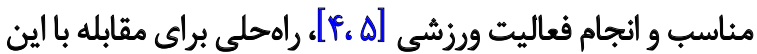
روند فرسايشى است.

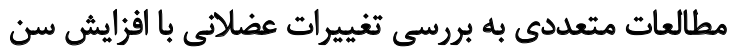

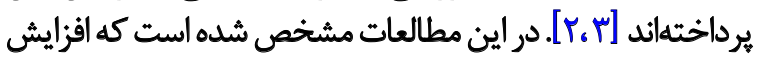

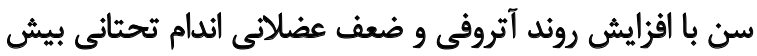

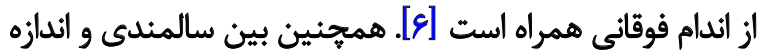

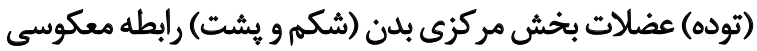

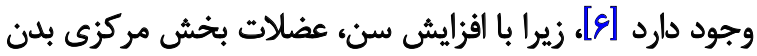

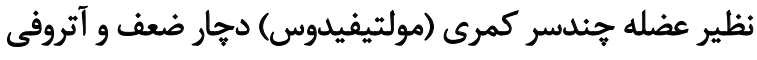

مقدمه سالمندى فرايندى جندبُعدى است و با كاهش بارز در عملكرد

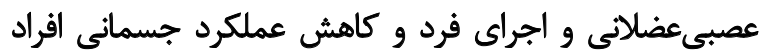

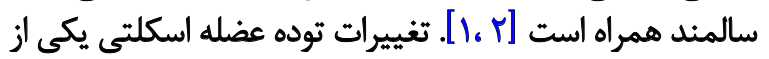

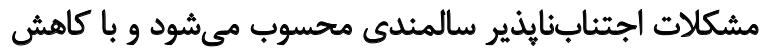

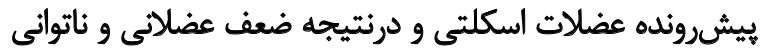

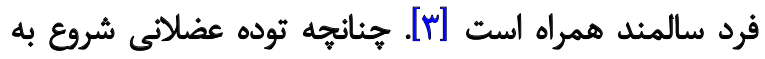

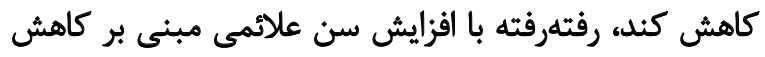

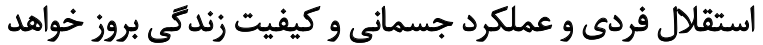

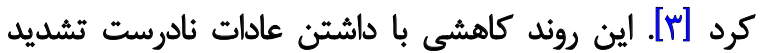

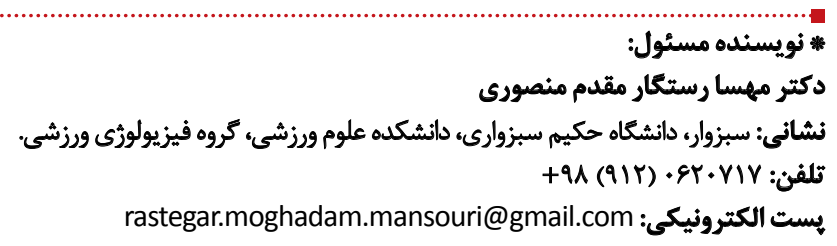


به بيمارى هاى ناتوان كنثده، سرطان و ناتوانى حركتى بود [19ا]

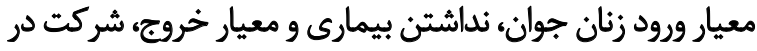

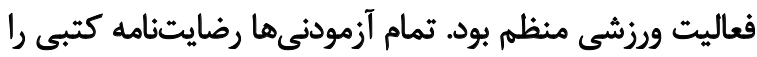

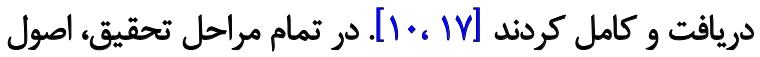

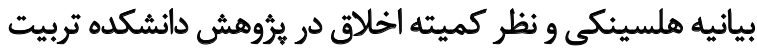

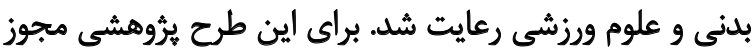

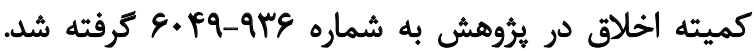

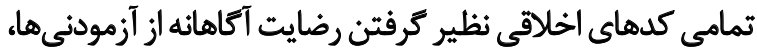

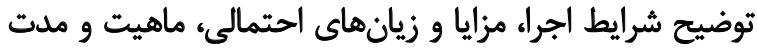

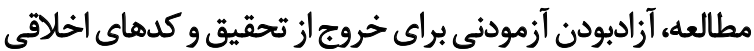

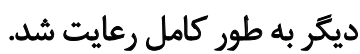

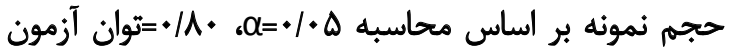

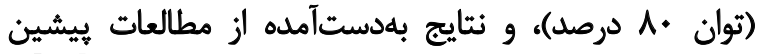

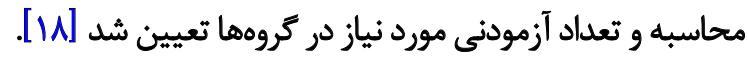
طبق محاسبات و با توان •^ درصد، 1 آزمودنى براى

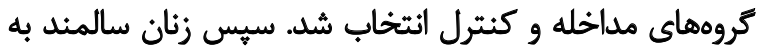

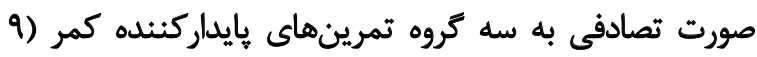

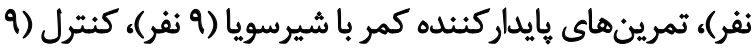

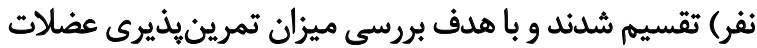

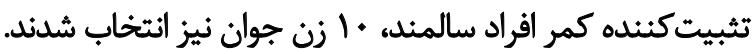

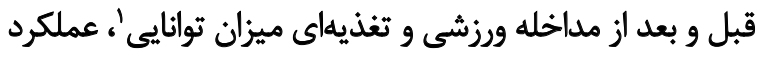

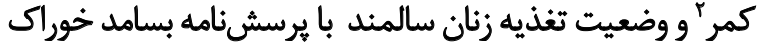

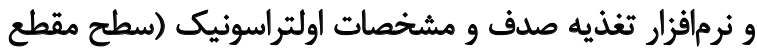

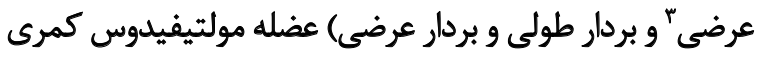

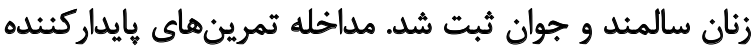

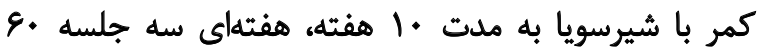

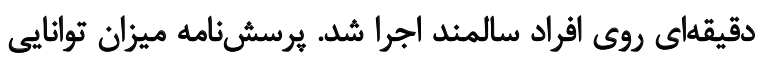

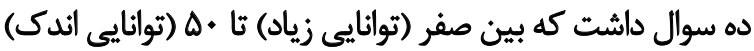

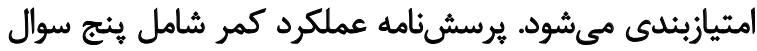

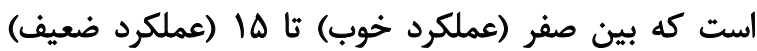

امتيازبندى مي كئ صود [19]

تمرينهايثر پايداركنتده كمر ازجمله تمرينهاى مقاومتى،

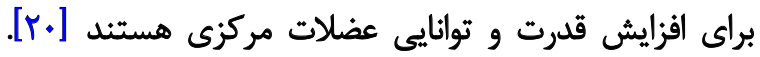

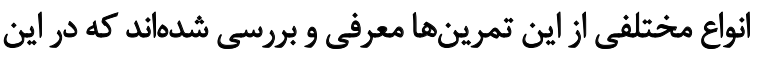

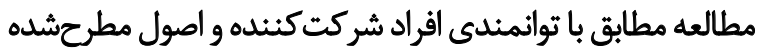

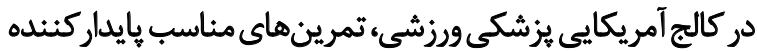

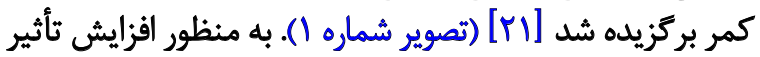

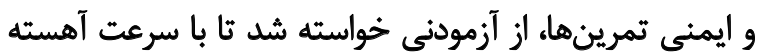

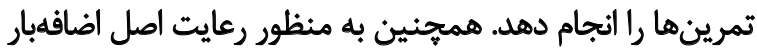

1. Oswestry Disability Index

2. Back Performance Scale

3. Cross Sectional Area
مىشود [V] و اين ضعف مى توائد با كاهش استقلال فردى همراه

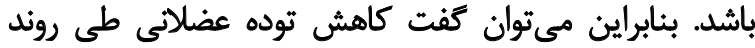

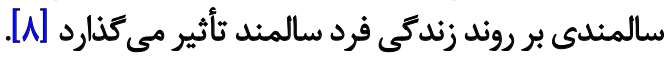
براى مقابله با تغيير توده عضلاتى و كاهش عملكرد جسمانى

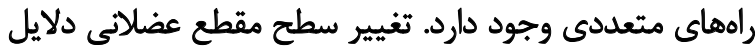

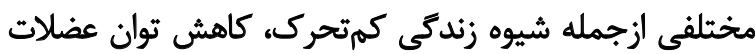

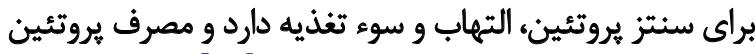

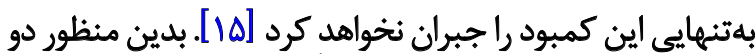

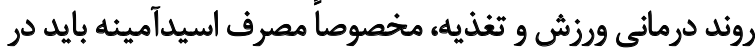

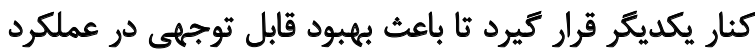

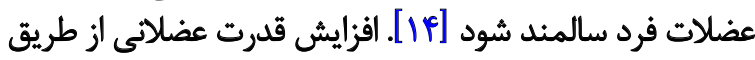

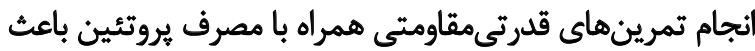

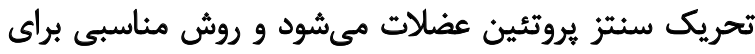

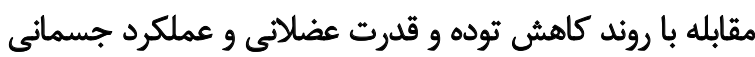

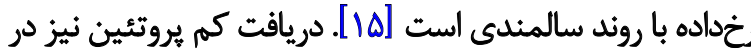

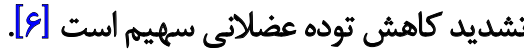

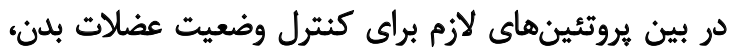

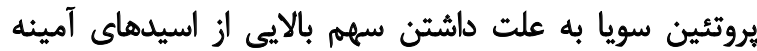

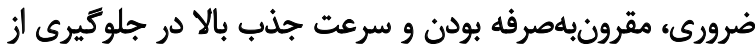

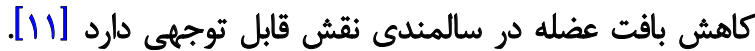

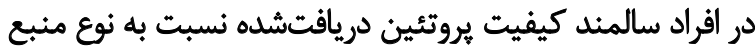

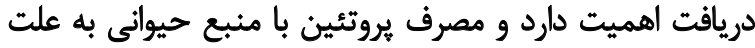

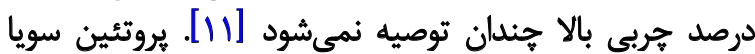

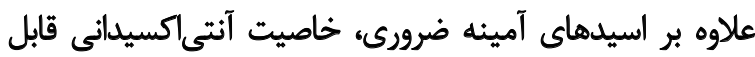

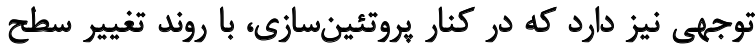

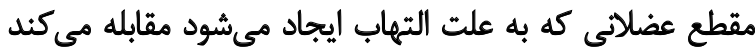

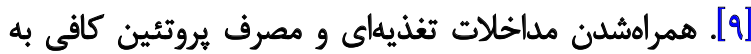

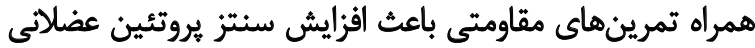

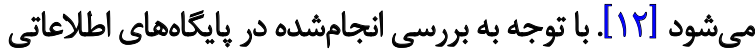

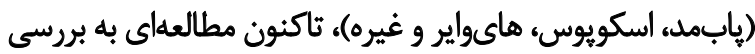

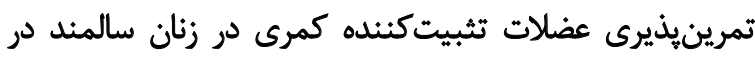

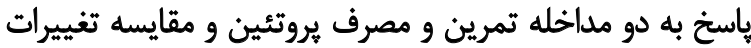

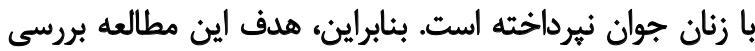

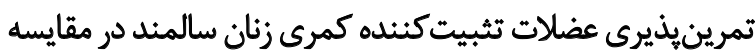

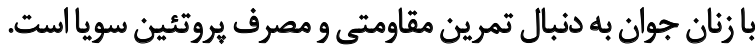

$$
\text { روش مطالعه }
$$

تحقيق حاضر از نوع نيمهتجربى بادو كروه سنى شركت كننده،

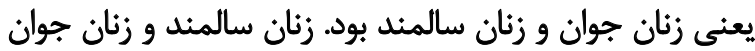

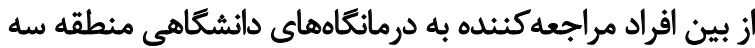

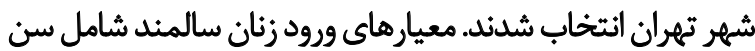

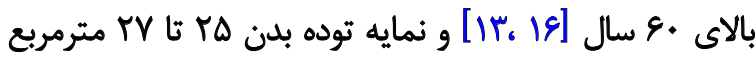

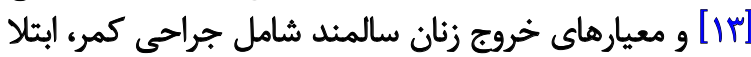



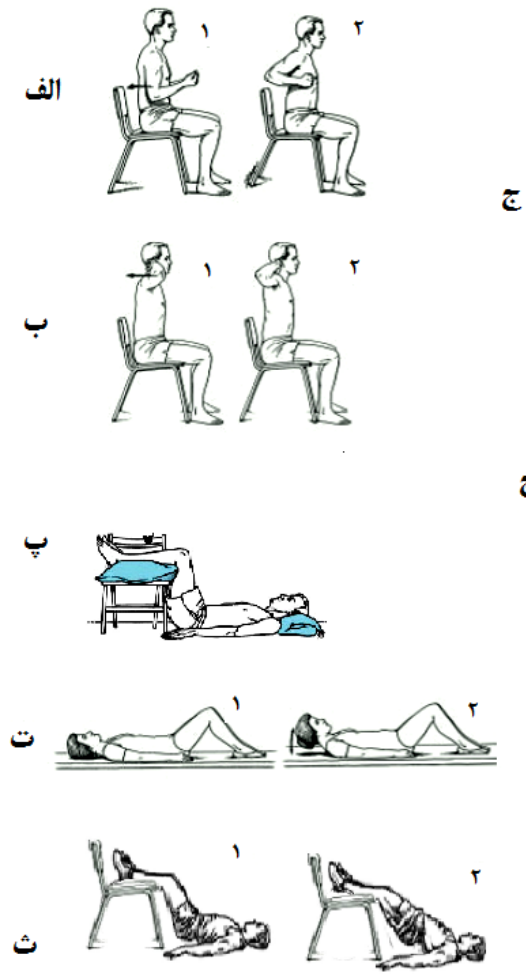
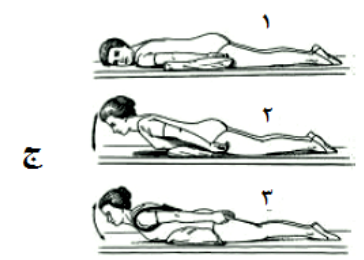

₹

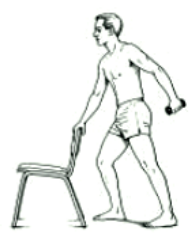

$\tau$

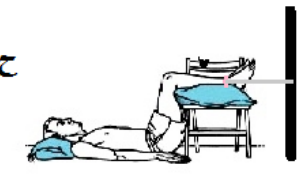

ill

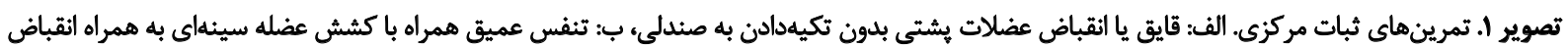

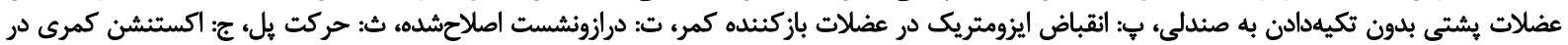

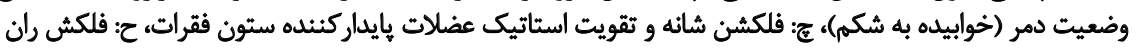

در دو هفته اول براى سازكارى و تقويت عضله مولتيفيدوس

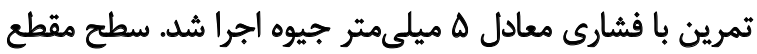

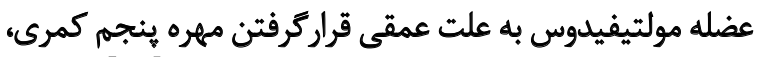

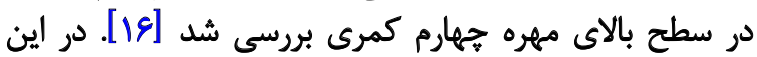

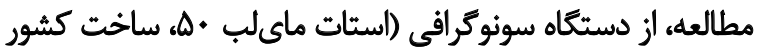

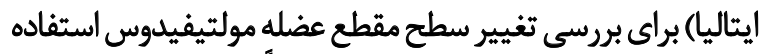

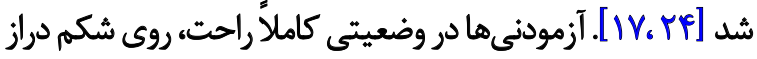

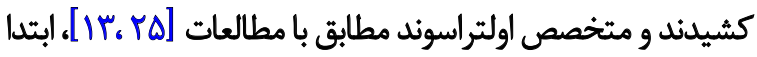

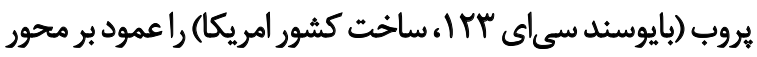

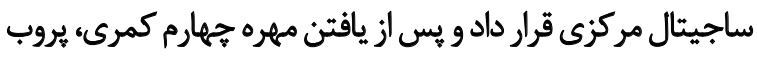

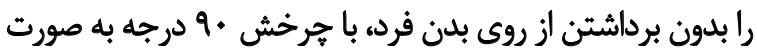
موازى با محور افقى قرار داد [بار] (تصوير شماره ب).

سطح مقطع عضله مولتيفيدوس از طريق دنبال كردن لبه ماربه

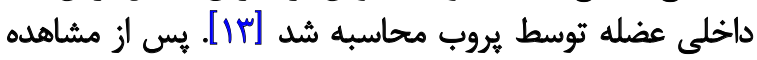

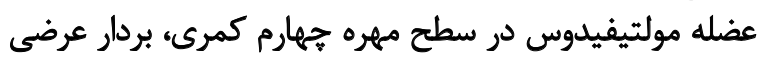

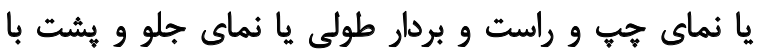

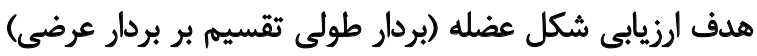

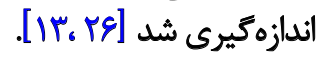
كروه تمرينهاى بايداركنيده كمر با شيرسويا يروتئين مصرفى
و سازكارى تدريجى از تراباند (شركت هايزئيك، ساخت كشور

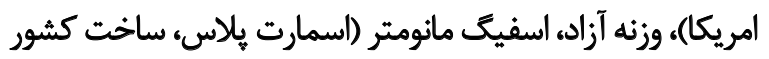

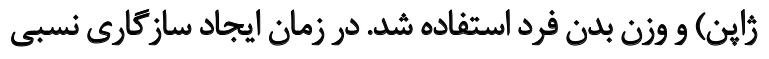

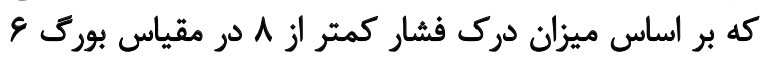

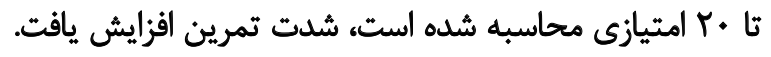

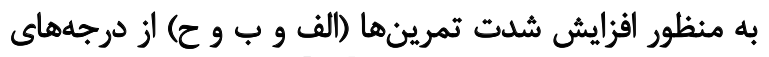

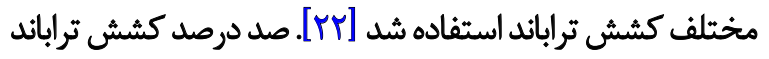

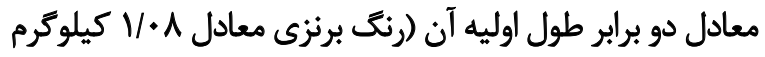
فشار) است (شركت هايرنيك، ساخت كشور آمريكا).

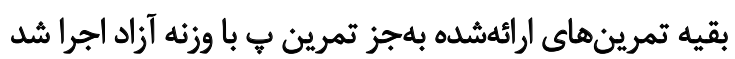

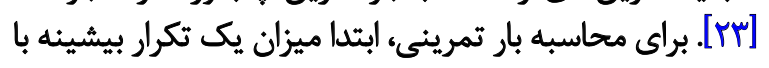

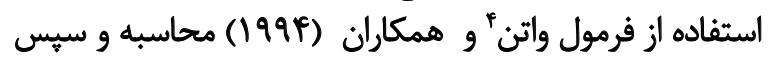

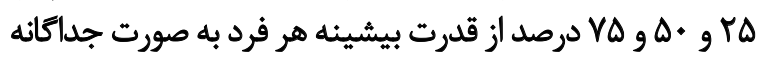

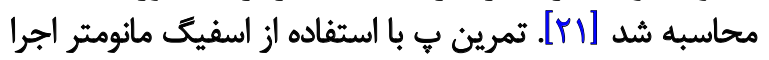

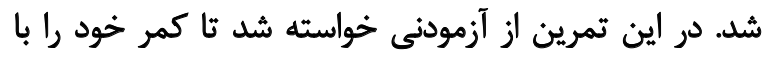

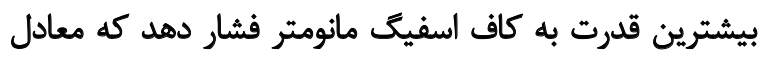

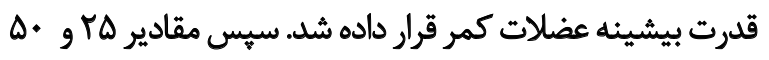

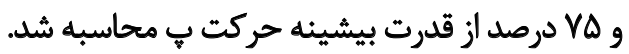



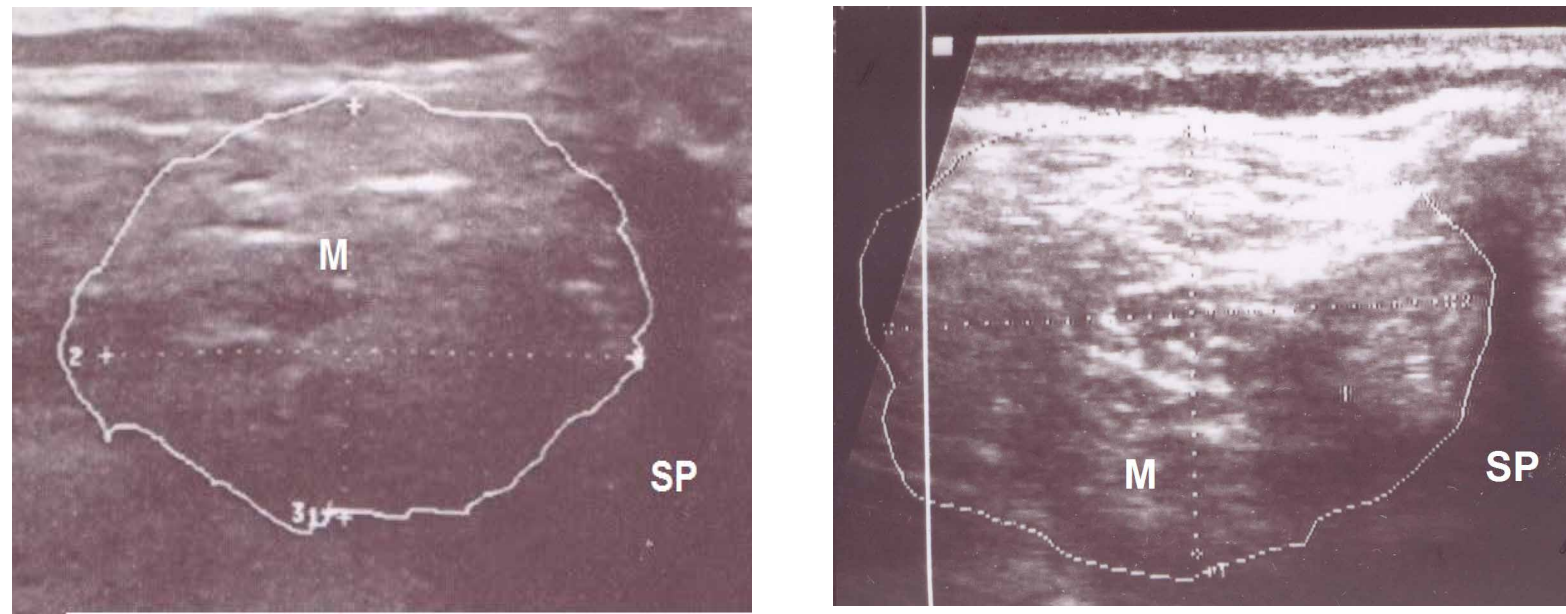

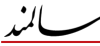

تصوير T. سطح مقطع عرضى عضله مولتيفيدوس با اولتراسوند در سطح مهره جهارم كمرى

:M مولتيفيدوس، SP زائده شوكى. تصوير سمت جبي: عضله مولتيفيدوس زنان مسن، تصوير سمت راست: عضله مولتيفيدوس زنان جوان

ميانكين سنى كيلوكرم و نمايه توده بدن

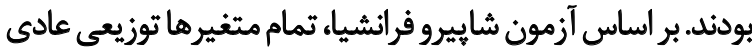

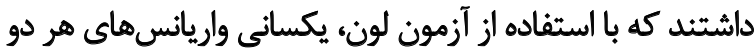

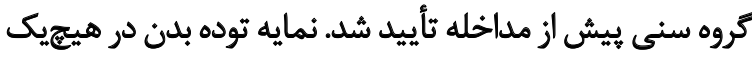

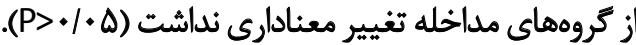

\section{بروسى عضله موالثيفيدوس}

در بررسى بين مروهى افراد جوان و سالمند بيش ازئ مداخله.

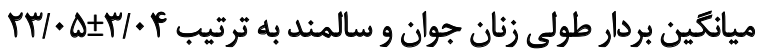

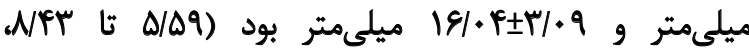

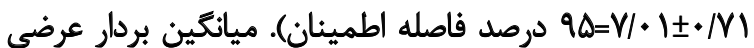

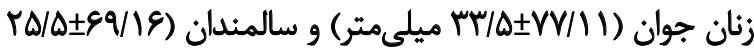

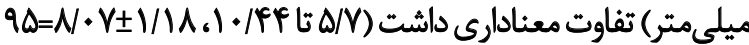

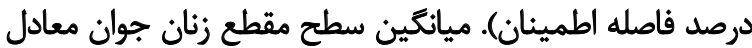

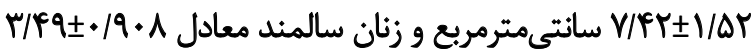

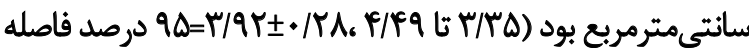

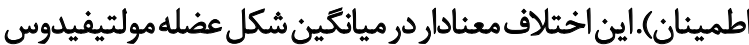

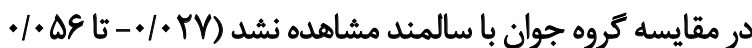

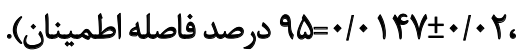

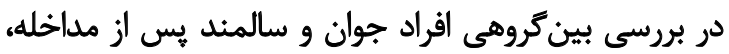

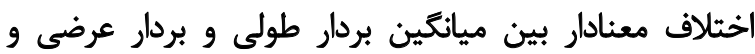

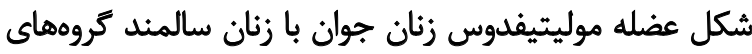

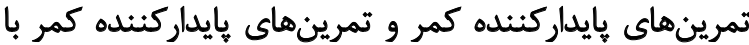

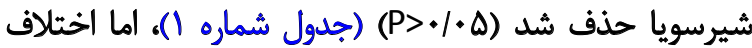

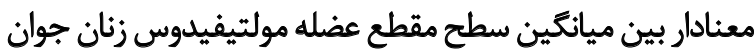

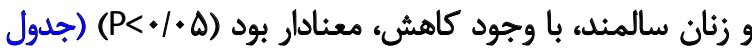

شيرسويا را به مدت · ا هفته، روزى سه وعده مصرف كردند. بر

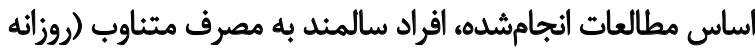

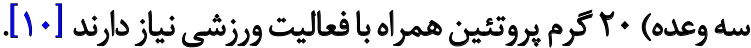

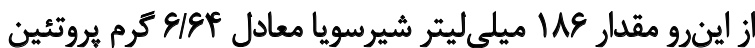

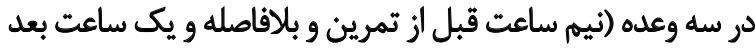

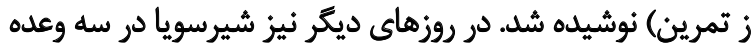

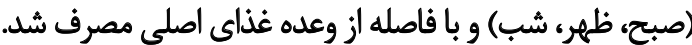

به منظور بررسى عادىبودن دادههاى قد و وزن إن و ونمايه توده

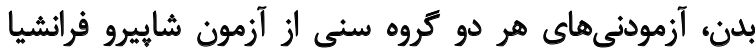

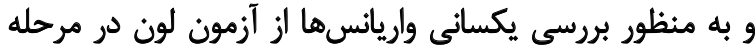

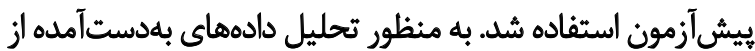

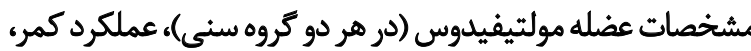

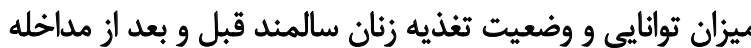

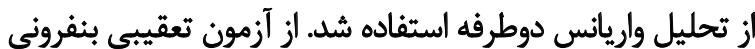

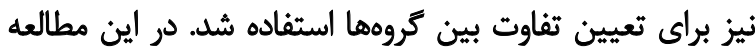

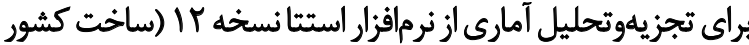
امريكا) و براي بررسى عضله مولتيفيدوس از نرمافزئرار ايمج.جى نسخه

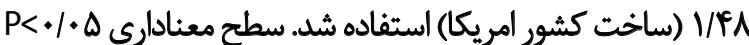
به عنوان ضابطه تصميم كيرى نتايج در نظر كرفته شد.

يافتهها

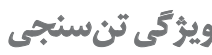

ييش از اجراى مداخله زنان سالمند شركت عنينده در

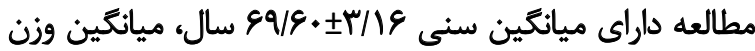

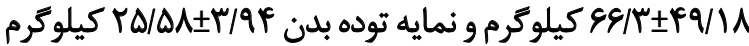

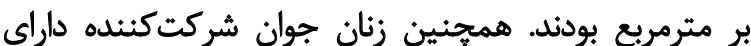




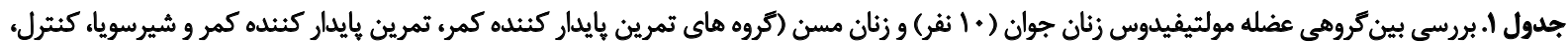

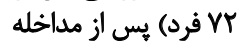

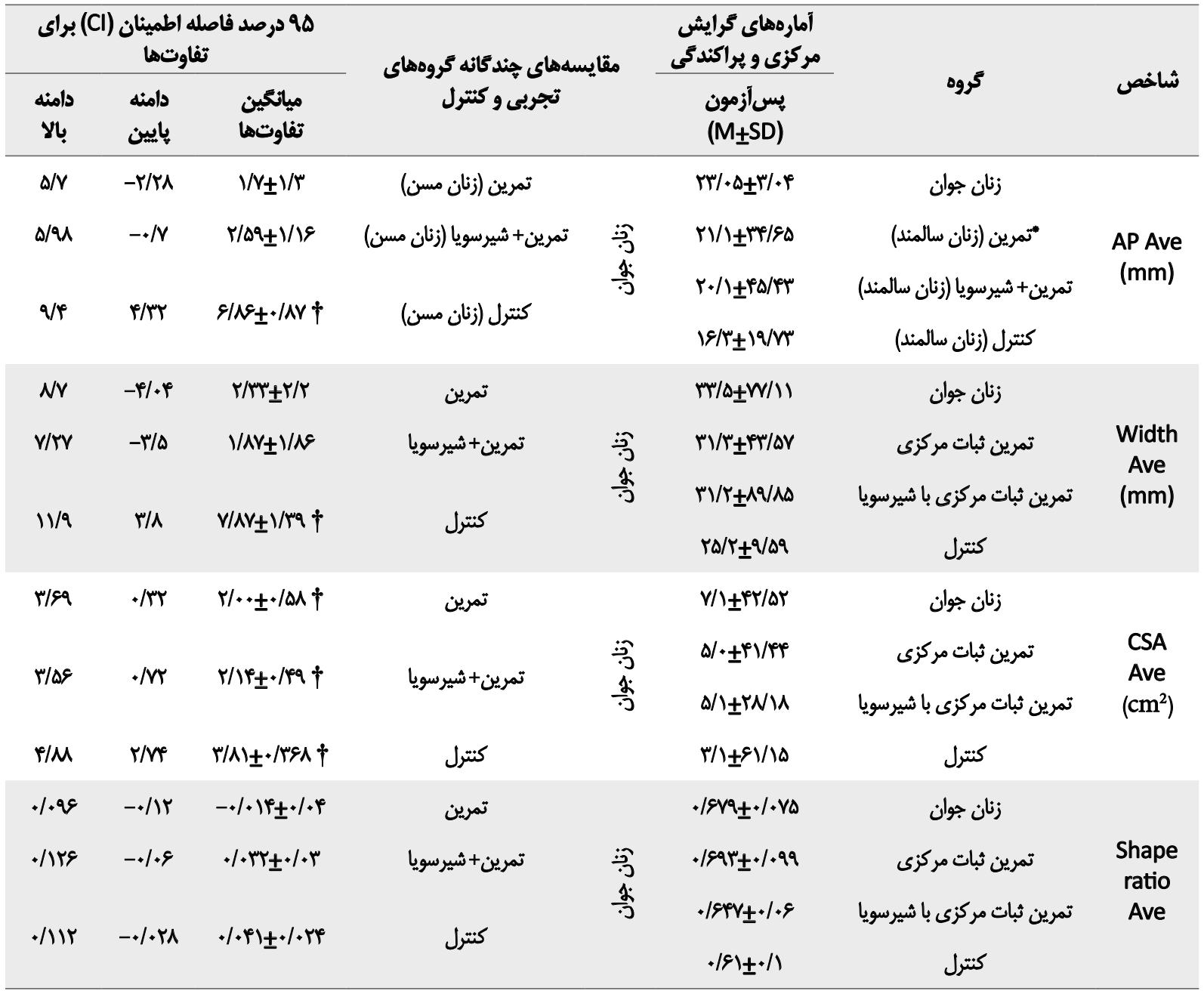

旔

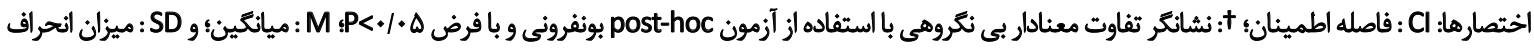
استاندارد كروه ملنظر تمرين: تمرين هايداركثنده كمر

\section{بروسى ميزان عملكرد كمر}

در بررسى بين كروهى عملكرد كمر، گروه تمرين بايداركنينده

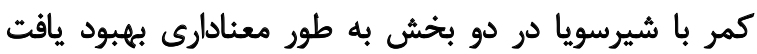

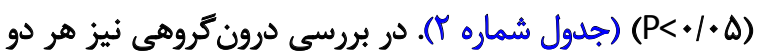

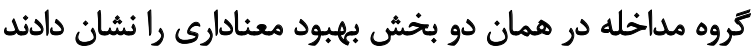

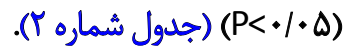

\section{وضعيت تغذيه}

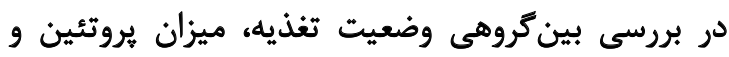

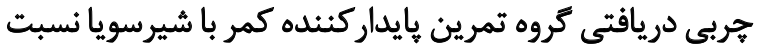

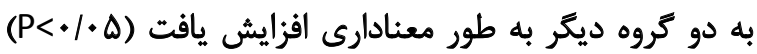

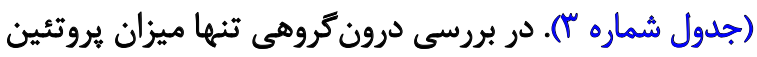

شماره (). در بررسى درون كروهى نيز ميانكين بردار طولى و بردار

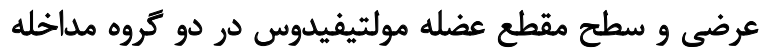

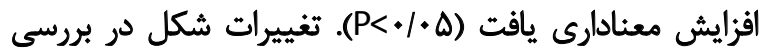

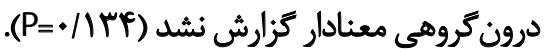

بورسى ميئان توانايى

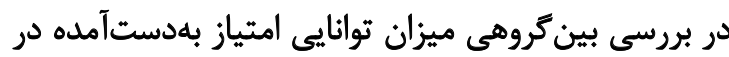

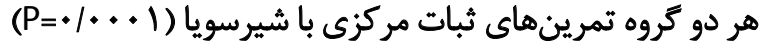

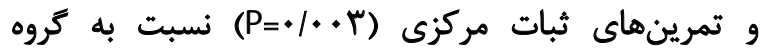

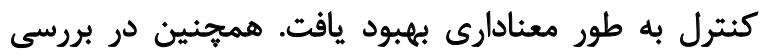

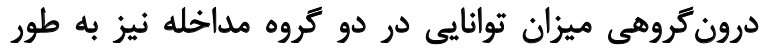
معنادارى بهبود يافت (تصوير شماره بان). 
هدف مطالعه حاضر بررسى تمرينيذيرى عضلات تثبيت كنينده

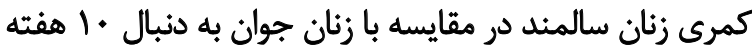

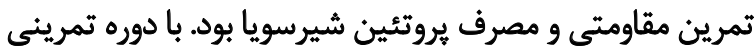

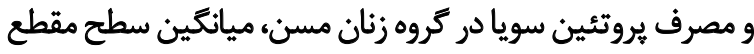

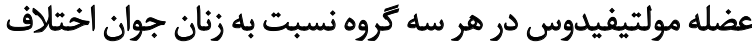

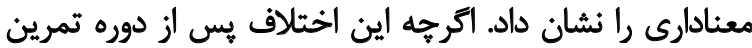

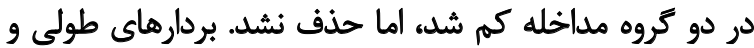

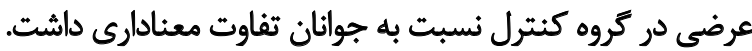

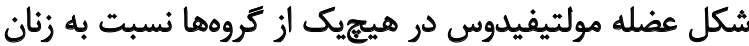

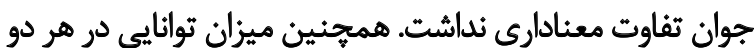

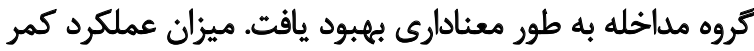

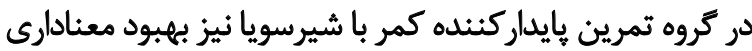

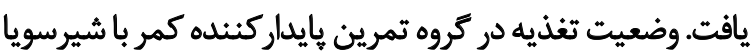
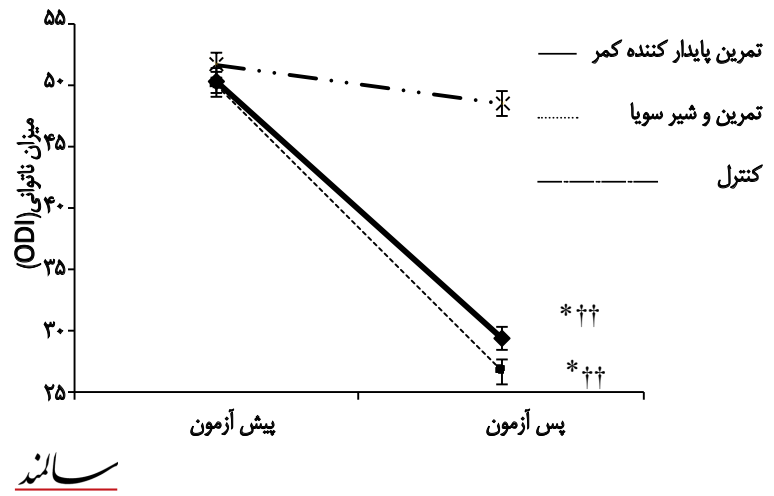

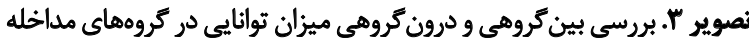

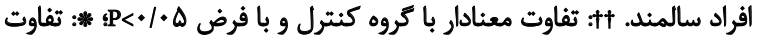

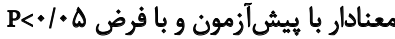

دريافتى كروه تمرين بايداركنيده كمر با شيرسويا افزايش

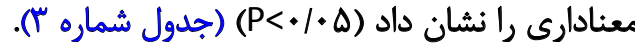
تغييرات معنادارى را نشان داد.

جدول r. بررسى بين كروهى و درون كروهى عملكرد كمر در زنان سالمند. كروهاي تمرين يايداركنيده كمر (9 فرد)، تمرين يايداركنيده كمر و شير سويا (9 فرد)،

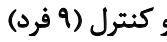

\begin{tabular}{|c|c|c|c|c|c|c|c|c|c|}
\hline \multicolumn{3}{|c|}{ هو دو تفاصله اطمينان (Cl) براى } & \multirow{2}{*}{\multicolumn{2}{|c|}{ 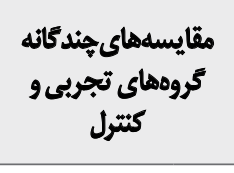 }} & \multirow{2}{*}{ معنادارى } & \multicolumn{2}{|c|}{ أمارههاى كرايش مركزى و } & \multirow[b]{2}{*}{ تروه } & \multirow[b]{2}{*}{ شلخص } \\
\hline بإمبالا & بايين & تفاوتهينا & & & & 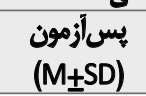 & $\begin{array}{l}\text { ييشآزمون } \\
(M \pm S D)\end{array}$ & & \\
\hline r/r & $-V+N e$ & $-\cdot / F \lambda_{ \pm} \cdot / r \cdot r$ & تمرين (زنان & & & & & & آزمون \\
\hline . & $-\cdot / N A$ & $-. / r \varphi_{ \pm}+/ r$ & تمرين+شيرسويا & 咨 & ع & $\begin{array}{l}W / T T_{ \pm} / A T^{\prime} \\
W \Delta_{ \pm} / Q S Q V\end{array}$ & $\begin{array}{l}V / \Delta \Delta_{ \pm} / / \\
V / \Delta \Delta \pm / 9 F\end{array}$ & كترين+شيرسويا (زلنان & ي جورشيلن \\
\hline זו/. &.$- / 04$ & $-\cdot / r \Delta_{ \pm} \cdot / M I$ & تمرين & & & $1 / \cdot \cdot \pm \cdot / 4$ & $V / \Delta \pm \cdot / \Lambda$ & تمرين & أزمون \\
\hline$+/ 11 f$ & $-+1 \Delta \cdot 1$ & $-* / 19 Y_{ \pm}+/ 19$ & تمرين+شيرسويا & 隽 & T & $\begin{array}{l}1 / 11 \pm \cdot / \pi \\
1 / \Delta \pm \cdot / \Delta 1\end{array}$ & 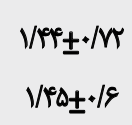 & تمرين tشيرسويا & كاغذازن \\
\hline . MIA & $-\cdot / A r$ & 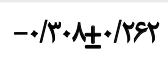 & تمرين & & & $\cdot V / / Y \pm V$ & $r / I V_{ \pm} /{ }^{\prime} u$ & تمرين & أَّمه: \\
\hline$-4 . \pi \varphi$ &.$- / 94$ & $-+/ R A_{ \pm} / T^{H t}$ & تمرين+شيرسويا & 䇰 &.$/ 1 \Delta$ & $\begin{array}{l}\cdot N A \pm \cdot / 9 \\
1 / 9 \Delta \pm \cdot / A r\end{array}$ & $\begin{array}{l}r / T \pm \pm / A T \\
Y / \pm \pm / N Q\end{array}$ & تمرين+شيرسويا & 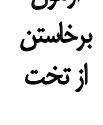 \\
\hline.$/ 4 \varphi$ & -.199 & $-\cdot / 1 V \Delta \pm \cdot / r \varepsilon$ & تمرين & & & $.\left|9 V_{ \pm} \cdot\right| \lambda \mid$ & $V / T \pm \pm V \cdot \cdot$ & تمرين & أزمون \\
\hline$\cdot / 1$ & - & $-. / M Y T \pm \cdot / T Y \varepsilon$ & تمرين+شيرسويا & 隽 & צ"א. & $\begin{array}{l}\cdot / / \pi \pm \cdot / \Delta \\
1 / \cdot \Delta \pm \cdot / \Delta 1\end{array}$ & 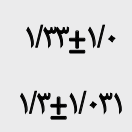 & تمرين+شيرسويا & 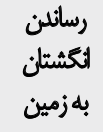 \\
\hline $.1 . \%$ &.$- / v a$ & $-. / / 4 \pm \pm / / u$ & تمرين & & & $\cdot V / \Delta \pm / \Delta t$ & $T / T \pm \pm / \Delta 1$ & تمرين & آزمون \\
\hline$-\cdot / N r$ &.$- / 1 n$ & $-. / r_{ \pm} \pm \cdot / N^{\dagger \dagger}$ & تمرين+شيرسويا & 䇰 & 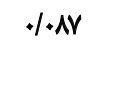 & $\begin{array}{l}\cdot V / \Delta \pm \pm / \Delta \\
r / T \Delta \pm / / A\end{array}$ & $\begin{array}{l}r / T_{ \pm} \cdot / \varphi \\
r / \pi \pm+/ P r\end{array}$ & تمرين بشيرسويا & جبلدكرن \\
\hline
\end{tabular}

证

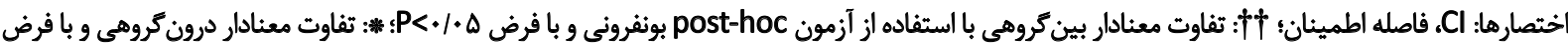

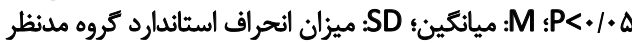


جدول لَ. بررسى بين كروهى و درون كروهى وضعيت تغذيه در زنان سالمند. كروهاي تمرين هايداركنئده كمر (9 فرد)، تمرين بايداركنئده كمر و شير سويا (9 فرد)،

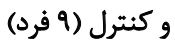

\begin{tabular}{|c|c|c|c|c|c|c|c|c|}
\hline \multicolumn{2}{|c|}{ ميانكين تفاوتهاو سطح معنادارى } & \multirow{2}{*}{\multicolumn{2}{|c|}{ 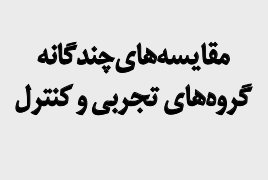 }} & \multirow{2}{*}{ معنادارى } & \multicolumn{2}{|c|}{ أمارههاى كرايش مركزي ويراكندكى } & \multirow{2}{*}{ كُوره } & \multirow{2}{*}{ شُاخص } \\
\hline معنادارى & تفاوتهان & & & & $\begin{array}{l}\text { بـ آزمون } \\
\left(M_{ \pm} S D\right)\end{array}$ & $\begin{array}{l}\text { بيشآزمون } \\
(M \pm S D)\end{array}$ & & \\
\hline.$/ 49$ & $A V \cdot r \pm 1 E / r$ & تمرين (زنان سالمند) & & & $M T T / V_{ \pm} M T / T$ & $\operatorname{MTQV/Q} \pm Q T / \Lambda$ & "تمرين (زنان سالمنل) & \\
\hline .181 & $V N \cdot \Delta \pm 1 F / \Delta$ & تمرين+شيرسويا & 羿 &.$/ 10$ & $\begin{array}{l}\text { MVNNE } \pm Q A / A \\
M T A \cdot / P \pm A Y / \cdot C\end{array}$ & 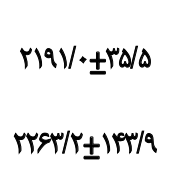 & كمرين+شيرسويا (زنان & $\begin{array}{c}\text { دريافتى Sل } \\
\text { (kcal) }\end{array}$ \\
\hline.$/ 014$ & $\begin{array}{l}r e / N \pm 1 \cdot / \varepsilon \Delta \\
-\Delta E N \pm N M q\end{array}$ & تمرين+شيرسوين & -3 & $\cdot / M^{e}$ & 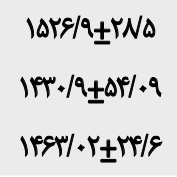 & 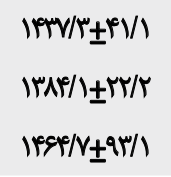 & تمرين+شيرين & $\begin{array}{c}\text { كربوهيلرات } \\
\text { (kcal) }\end{array}$ \\
\hline.$/ 4 T A$ & 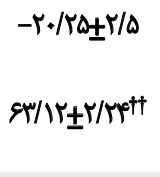 & تمرين+شيرسويا & $\stackrel{3}{3}$ & $+1 *+1^{\dagger}$ & 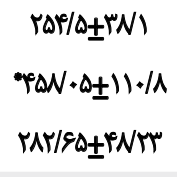 & 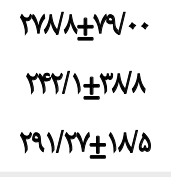 & تمرين+شيرسويا & $\begin{array}{l}\text { بروتئين } \\
\text { (kcal) }\end{array}$ \\
\hline$\circ / \circ \Delta V$ & 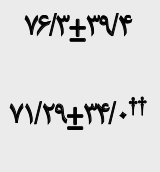 & تمرين+شيرسويا & $\rightarrow$ & $.1 . . .1^{\dagger}$ & 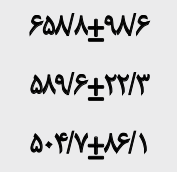 & $\begin{array}{l}\Delta \cdot \Delta / Y \pm T r / \Delta \\
\Delta \&+/ A \pm q \cdot / \varepsilon \\
\Delta \cdot V / V Q \pm T r / T \&\end{array}$ & تمرين+شيرسويا & $\begin{array}{l}\text { (kcal) } \\
\text { (kella }\end{array}$ \\
\hline
\end{tabular}

故

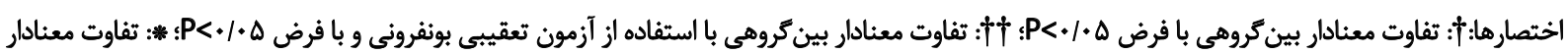

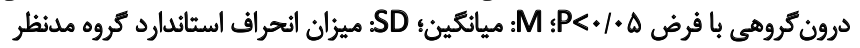

سويا نسبت به شير تأثيرى بر مسيرهاى يروتئولتيك نداشته

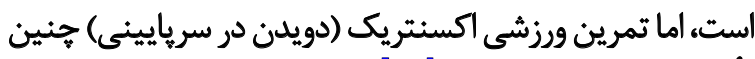
تأثيرى را دربر داشته است است استرين [11].

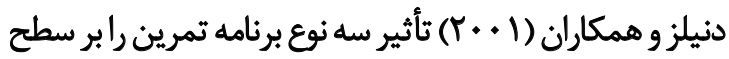

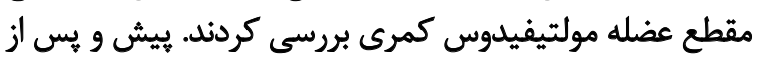
مطالعه سطح مقطع عضله مولتيفيدوس كمرى تمري تمام آزمودنى برديها

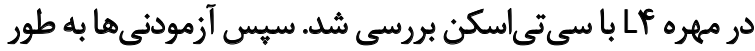

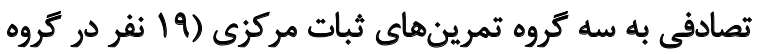

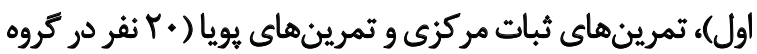

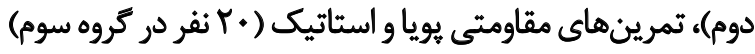

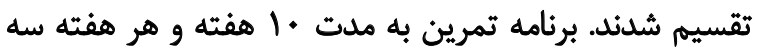

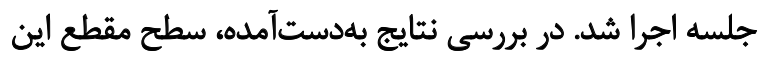

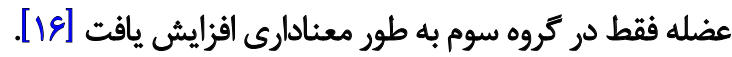
در بررسى علت تغييرات عضله مولتيفيدوس و بهبود ميزان

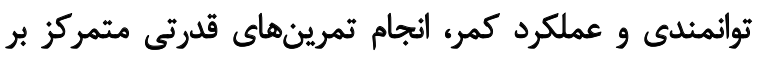

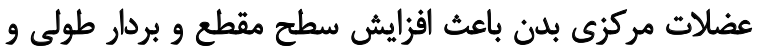

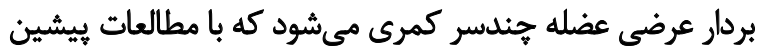

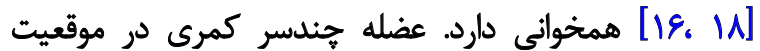

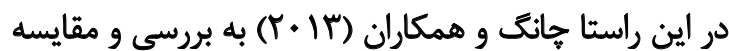

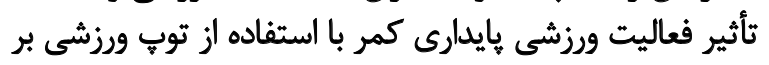

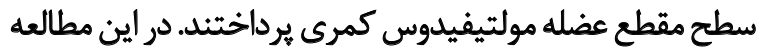

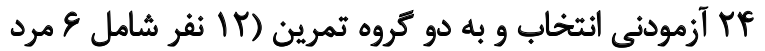

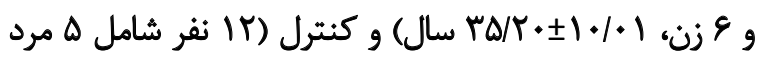

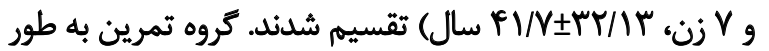

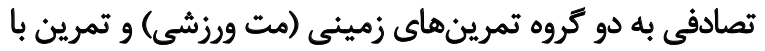

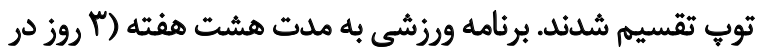

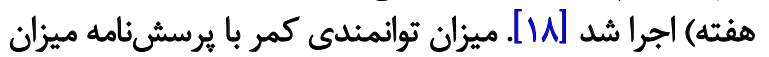

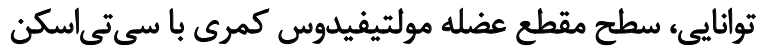

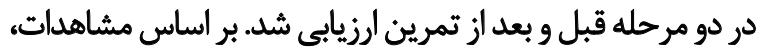

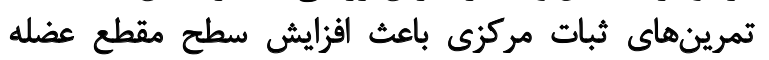

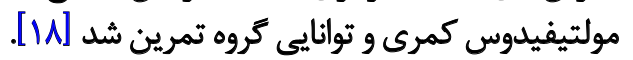

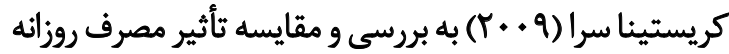

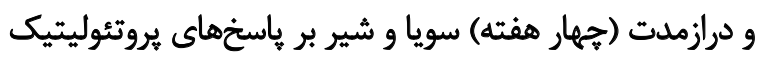

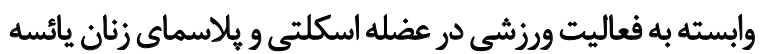

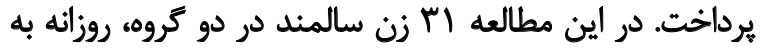

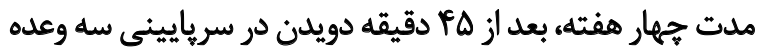

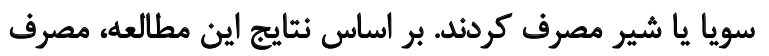


به افراد جوان به علت مقاومت آنابوليكه و اختلال در مسيرهاى

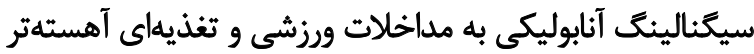

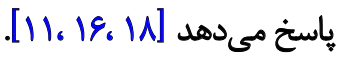

در بررسى انواع تمرينهاى قدرتى، انجام تمرينهاى ثبات

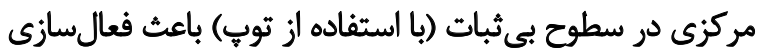

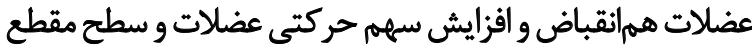

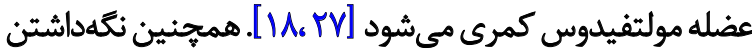

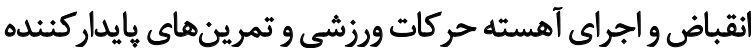

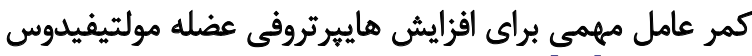

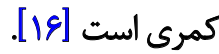

\section{نتيجلكَيرى نُهايي}

انجام ده هفته مداخله تمرينهاى يايداركنئده كمر و مصرف

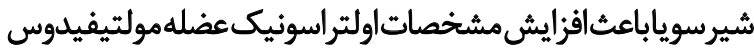

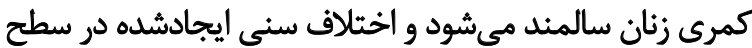

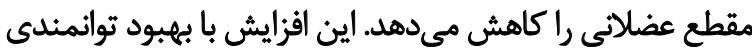

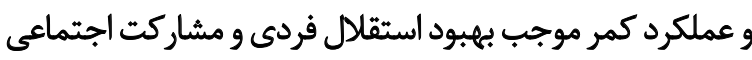

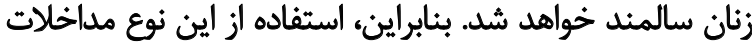
براي بهبود وضعيت عملكرد زنان سالمئد جامعه توصيه مي نئنود.

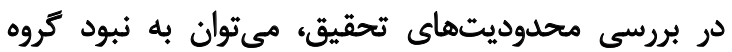

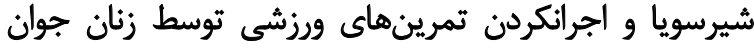

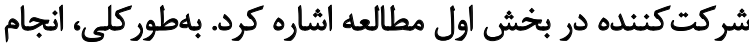

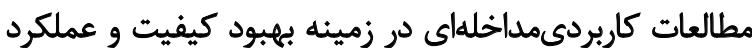

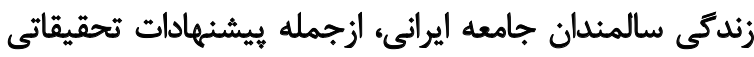

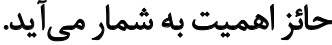

$$
\text { تشكر و قدودائى }
$$

اين مقاله از إياينامه مقطع دكتراي خانم مهسا رستكار مقدم

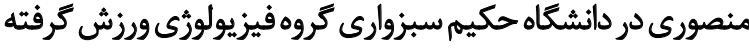

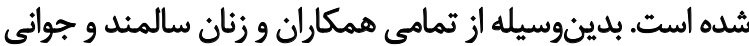
كه ما را در اجراي اين تحقيق يارى كردند قدردانى مي مئينيم.
آناتوميكى محدودى قرار كرفته است؛ بهطورى كه از اطراف با زوايد

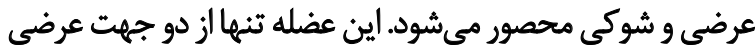

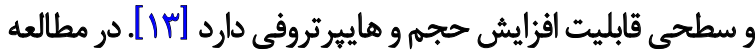

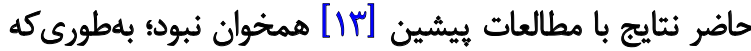

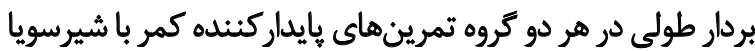

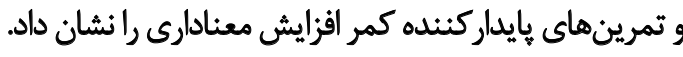
بردار عرضى فقط در كروه تمرينهاى بايداركنيده كمر باشيرسويا

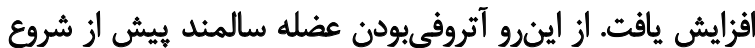

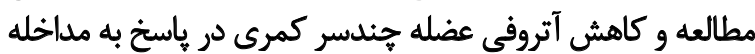

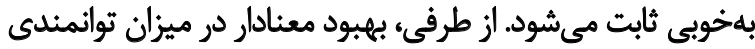

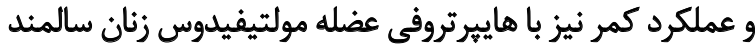

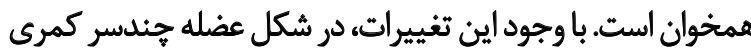

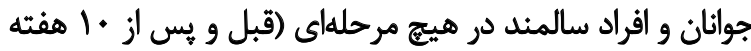

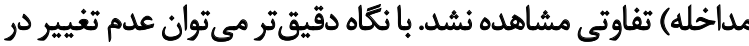

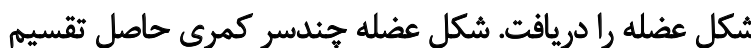

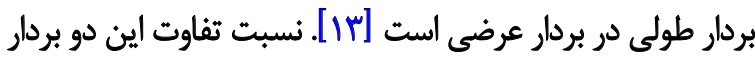

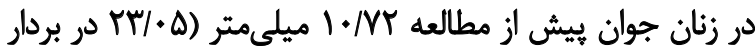
طولى و د

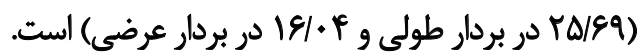

همجينين باوجود افزايش اين دو بردار در گروه هاي تمرينهاي

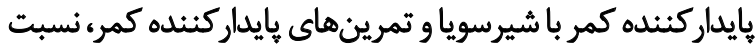

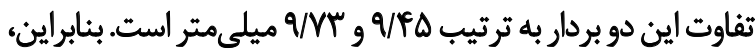

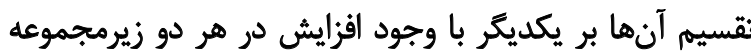

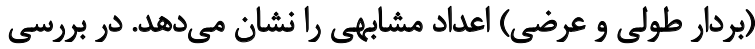

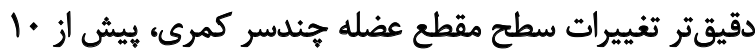

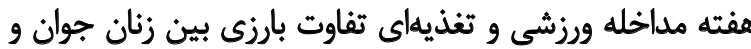

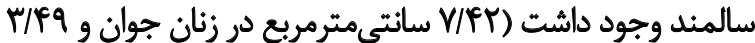

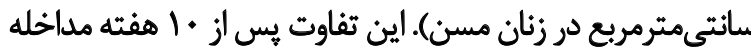

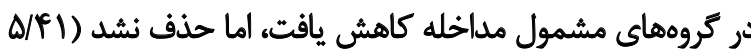

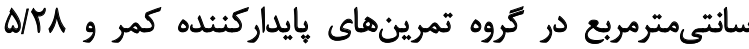
سانتىمترمربع در كروه تمرينهاي بايداركنينده كمر باشيرسويا). نكته جالب توجه مشابهشدن سطح مقطع زنان سان سالمند

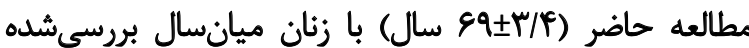

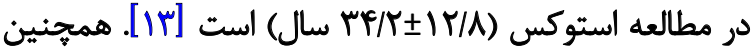

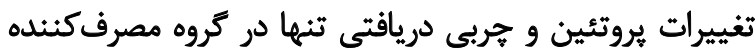

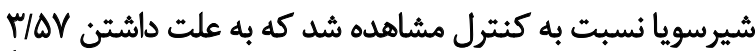

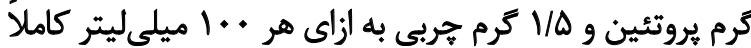

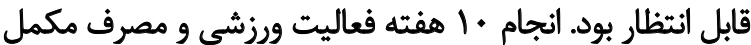

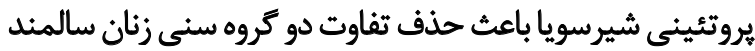

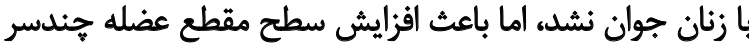

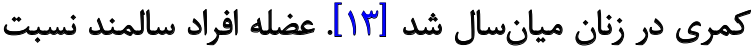




\section{References}

[1] Seene T, Kaasik P. Muscle weakness in the elderly: Role of sarcopenia, dynapenia, and possibilities for rehabilitation. European Review of Aging and Physical Activity. 2012; 9(2):109-17. doi: $10.1007 /$ s11556-012-0102-8

[2] Evans WJ. Skeletal muscle loss: Cachexia, sarcopenia, and inactivity. American Journal of Clinical Nutrition. 2010; 91(4):1123-7. doi: 10.3945/ajcn.2010.28608a

[3] Lynch GS. Sarcopenia - Age related muscle wasting and weakness. Heidelberg: Springer; 2011. doi: 10.1007/978-90-481-9713-2

[4] Beas-Jiménez JD, López-Lluch G, Sánchez-Martínez I, MuroJiménez A, Rodríguez-Bies E, Navas P. [Sarcopenia: Implications of physical exercise in its pathophysiology, prevention and treatment (Spanish)]. Andalusian Journal of Sports Medicine. 2011; 4(4):158-66.

[5] Greig CA. Nutritional approaches to the management of sarcopenia. Nutrition Bulletin. 2013; 38(3):344-8. doi: 10.1111/nbu.12046

[6] Ikezoe T, Mori N, Nakamura M, Ichihashi N. Effects of age and inactivity due to prolonged bed rest on atrophy of trunk muscles. Euro-pean Journal of Applied Physiology. 2011; 112(1):43-8. doi: 10.1007/s00421-011-1952-x

[7] Ward SR, Kim CW, Eng CM, Gottschalk LJ, Tomiya A, Garfin SR, et al. Architectural analysis and intraoperative measurements demon-strate the unique design of the multifidus muscle for lumbar spine stability. The Journal of Bone and Joint Surgery-American Volume. 2009; 91(1):176-85. doi: 10.2106/jbjs.g.01311

[8] Rizzoli R, Reginster JY, Arnal JF, Bautmans I, Beaudart C, Bischoff-Ferrari $\mathrm{H}$, et al. Quality of life in sarcopenia and frailty. Calcified Tissue International. 2013; 93(2):101-20. doi: 10.1007/s00223013-9758-y

[9] Serra MC, Beavers KM, Beavers DP, Willoughby DS. Effects of 28 days of dairy or soy ingestion on skeletal markers of inflammation and proteolysis in post-menopausal women. Nutrition and Health. 2012; 21(2):117-30. doi: 10.1177/0260106012467243

[10] Nedergaard A, Henriksen K, Karsdal MA, Christiansen C. Musculoskeletal ageing and primary prevention. Best Practice \& Research Clin-ical Obstetrics \& Gynaecology. 2013; 27(5):673-88. doi: 10.1016/j.bpobgyn.2013.06.001

[11] Serra MC. Effects of four weeks of daily soy or dairy milk ingestion on the exercise-induced proteolytic responses in plasma and skeletal muscle in a post-menopausal female population $[\mathrm{PhD}$ thesis]. Texas: Baylor University; 2009.

[12] Candow DG, Forbes SC, Little JP, Cornish SM, Pinkoski C, Chilibeck PD. Effect of nutritional interventions and resistance exercise on ag-ing muscle mass and strength. Biogerontology. 2012 13(4):345-58. doi: 10.1007/s10522-012-9385-4

[13] Stokes M, Rankin G, Newham DJ. Ultrasound imaging of lumbar multifidus muscle: Normal reference ranges for measurements and prac-tical guidance on the technique. Manual Therapy. 2005; 10(2):116-26. doi: 10.1016/j.math.2004.08.013

[14] Brooks NE, Cadena SM, Vannier E, Cloutier G, Carambula S, Myburgh $\mathrm{KH}$, et al. Effects of resistance exercise combined with essential amino acid supplementation and energy deficit on markers of skeletal muscle atrophy and regeneration during bed rest and active recov-ery. Muscle \& Nerve. 2010; 42(6):927-35. doi 10.1002/mus. 21780
[15] Breen L, Phillips SM. Interactions between exercise and nutrition to prevent muscle waste during aging. British Journal of Clinical Phar-macology. 2012; 75(3):708-15. doi: 10.1111/j.13652125.2012.04456.x

[16] Danneels LA. Effects of three different training modalities on the cross sectional area of the lumbar multifidus muscle in patients with chronic low back pain. British Journal of Sports Medicine. 2001; 35(3):186-91. doi: 10.1136/bjsm.35.3.186

[17] Cruz-Jentoft AJ, Baeyens JP, Bauer JM, Boirie Y, Cederholm T, Landi F, et al. Sarcopenia: European consensus on definition and diagnosis: Report of the European working group on sarcopenia in older people. Age and Ageing. 2010;39(4):412-23. doi:10.1093/ ageing/afq034

[18] Chung S, Lee J, Yoon J. Effects of stabilization exercise using a ball on mutifidus cross-sectional area in patients with chronic low back pain. Journal of Sports Science and Medicine. 2013; 12(3):53341. PMCID: PMC3772599

[19] Hawker GA, Mian S, Kendzerska T, French M. Measures of adult pain: Visual analog scale for pain, numeric rating scale for pain, McGill pain questionnaire, short-form McGill pain questionnaire, chronic pain grade scale, short form-36 bodily pain scale. Arthritis Care \& Research. 2011; 63(11):240-52. doi: 10.1002/ acr.20543

[20] Sinaki M. [Musculoskeletal rehabilitation in patients with osteoporosis-rehabilitation of osteoporosis program-exercise (German)]. Journal für Mineralstoffwechsel \& Muskuloskelettale Erkrankungen. 2010; 17(2):60-5.

[21] Ratamess NA. ACSM's foundations of strength training and conditioning. Philadelphia: Lippincott Williams \& Wilkins; 2012.

[22] Patterson RM, Stegink Jansen CW, Hogan HA, Nassif MD. Material properties of Thera-Band tubing. Physical Therapy. 2001; 81(8):1437-45. doi: 10.1093/ptj/81.8.1437

[23] American College of Sports Medicine Position Stand. Progression models in resistance training for healthy adults. Medicine \& Science in Sports \& Exercise. 2009; 41(3):687-708. doi: 10.1249/ mss.0b013e3181915670

[24] Cesari M, Fielding RA, Pahor M, Goodpaster B, Hellerstein M, Van Kan GA, et al. Biomarkers of sarcopenia in clinical trials-recommendations from the international working group on sarcopenia. Journal of Cachexia, Sarcopenia and Muscle. 2012; 3(3):181-90. doi: 10.1007/s13539-012-0078-2

[25] Koppenhaver SL, Hebert JJ, Fritz JM, Parent EC, Teyhen DS, Magel JS. Reliability of rehabilitative ultrasound imaging of the transversus abdominis and lumbar multifidus muscles. Archives of Physical Medicine and Rehabilitation. 2009; 90(1):87-94. doi: 10.1016/j.apmr.2008.06.022

[26] Hides JA, Cooper DH, Stokes MJ. Diagnostic ultrasound imaging for measurement of the lumbar multifidus muscle in normal young adults. Physiotherapy Theory and Practice. 1992; 8(1):1926. doi: 10.3109/09593989209108076

[27] Carter JM, Beam WC, McMahan SG, Barr ML, Brown LE. The effects of stability ball training on spinal stability in sedentary individuals. Journal of Strength and Conditioning Research. 2006; 20(2):429-35. doi: 10.1519/00124278-200605000-00032 